\title{
Photon self-energy in a magnetized chiral plasma from kinetic theory
}

\author{
Han Gao®, ${ }^{*}$ Zonglin Mo, ${ }^{\dagger}$ and Shu Lin \\ School of Physics and Astronomy, Sun Yat-Sen University, Zhuhai 519082, China
}

(Received 23 February 2020; accepted 22 June 2020; published 8 July 2020)

\begin{abstract}
We study the photon self-energy in magnetized chiral plasma by solving the response of electromagnetic field perturbations in chiral kinetic theory with Landau level states. With lowest Landau level approximation and in the collisionless limit, we find solutions for three particular perturbations: the parallel electric, static perpendicular electric field, and static perpendicular magnetic field, corresponding to chiral magnetic wave, drift state, and tilted state, from which we extract components of photon self-energy in different kinematics. We that show no solution is possible for more general field perturbations. We argue that this is an artifact of the collisionless limit: while static solutions corresponding to the drift state and tilted state can be found, they cannot be realized dynamically without interaction between Landau levels. We also discuss the possible manifestation of side-jump effects due to both boost and rotation, with the latter due to the presence of background magnetic field.
\end{abstract}

DOI: 10.1103/PhysRevD.102.014011

\section{INTRODUCTION}

There has been a long history of work towards an understanding of vacuum polarization by electromagnetic fields. The full effective action of vacuum for an arbitrary constant electromagnetic field was established by Heisenberg and Euler [1], who predicted the critical electric field in vacuum. It was later realized by Schwinger [2] that the critical electric field leads to pair production. On the other hand, while the magnetic field does not destabilize the vacuum, it does modify vacuum properties, such as enhancing the pair production rate [3] and causing vacuum birefringence [4-6]; see [7,8] for recent reviews.

Recently there has been growing interest in the effect of the magnetic field in the chiral medium in a variety of systems including quark-gluon plasma, Weyl semimetal, etc. The magnetic field in the chiral medium is known to lead to novel anomalous transport such as the chiral magnetic effect [9-12], chiral separation effect [13,14], chiral magnetic wave [15], etc. Furthermore, the presence of magnetic field also modifies existing transport coefficient-like conductivities [16-23] and viscosities [24,25] nontrivially. In the regime of the linear response, these transport phenomena are characterized by photon self-energy in the magnetized

\footnotetext{
"gaoh26@mail2.sysu.edu.cn mozlin@mail2.sysu.edu.cn

†inshu8@mail.sysu.edu.cn
}

Published by the American Physical Society under the terms of the Creative Commons Attribution 4.0 International license. Further distribution of this work must maintain attribution to the author(s) and the published article's title, journal citation, and DOI. Funded by SCOAP. chiral medium. In the presence of the magnetic field, the photon self-energy contains a very rich structure and is also very complicated in general. There have been many field theoretic attempts to study the photon self-energy [26-29]; see also studies on gluon self-energy [30,31].

A distinguishing feature of the chiral fermion from the classical particle is its spin, which is a genuine quantum quantity measured in $\hbar$. A semi-classical expansion in $\hbar$ gives rise to the chiral kinetic theory (CKT) [32-57]. It has been successfully applied to study the transport phenomena of the chiral medium in response to the weak electromagnetic field [58-61], where each power of electromagnetic field contributes $O(\hbar)$. In the regime of the strong magnetic field, a different expansion scheme is used giving rise to a chiral kinetic theory based on Landau level (LL) states $[18,22,62]$. The purpose of this paper is to apply this chiral kinetic theory to study the photon self-energy in magnetized chiral plasma as an alternative approach to the selfenergy problem. For simplicity, we work in the strong magnetic field and collisionless limit. We will reproduce the field theoretic results to the accuracy of the CKT approach and show some new results for the drift state and tilted state.

The paper is organized as follows: in Sec. II, we summarize generalities of photon self-energy, in Sec. III, we give a short review of chiral kinetic equations based on LL states and analyze the structure of equations, and in Sec. IV, we present solutions corresponding to three specific perturbations and discuss the physical implications of them. We further show it is not possible to obtain more solutions for more general perturbations. We will argue that it is an artifact of the collisionless limit; in Sec. V we summarize the results and discuss future directions. 
Throughout this paper, we primarily study the chiral medium consisting of right-handed fermions with charge $Q=|e|$. Contribution of left-handed fermions will be added when comparing with field theoretic results. For simplicity, we set $e=1$ and reinstate it in the end. We use the following nonstandard convention $p_{\mu}=\left(p_{0}, p_{1}, p_{2}, p_{3}\right)$ for convenience. The magnetic field points in the $x_{3}$ direction.

\section{GENERALITIES OF PHOTON SELF-ENERGY IN A MAGNETIZED MEDIUM}

The photon self-energy in the imaginary time formalism is defined by [63]

$$
\Pi_{E}^{\mu \nu}\left(x, x^{\prime}\right)=\left\langle\hat{T}\left(J_{E}^{\mu}(x) J_{E}^{\nu}\left(x^{\prime}\right)\right)\right\rangle=\frac{\delta J_{E}^{\mu}(x)}{\delta A_{\nu}^{E}\left(x^{\prime}\right)},
$$

where $\hat{T}$ denotes time ordering in Euclidean time. We can rewrite (1) in a simpler form in momentum space:

$$
\delta J_{E}^{\mu}(q)=\Pi_{E}^{\mu \nu}(q) \delta A_{\nu}^{E}(q) .
$$

Note that the Euclidean frequency $q_{4}$ takes discrete values of Matsubara frequencies $2 \pi n T$, with $T$ being temperature. It can be analytically continued to the complex frequency plane. Taking $q_{4} \rightarrow i\left(q_{0}+i \epsilon\right)$, we obtain the more useful retarded photon self-energy

$$
\delta J^{\mu}(q)=\Pi_{R}^{\mu \nu}(q) \delta A_{\nu}(q),
$$

with $J_{E}^{4}(q) \rightarrow i J^{0}(q)$ and $A_{E}^{4}(q) \rightarrow i A^{0}(q)$. Equation (3) expresses the current as a response to external electromagnetic field perturbation, which can be studied in kinetic theory. We will mainly study retarded self-energy in the paper.

For a parity breaking chiral medium consisting of righthanded fermions, $\Pi_{R}^{\mu \nu}$ is, in general, not symmetric in the Lorentz indices. Nevertheless, $\Pi_{R}^{\mu \nu}$ is still constrained by an anomalous Ward identity. To derive an anomalous Ward identity in the regime of the strong magnetic field, we note that there is an effective dimensional reduction from $3+1 D$ to $1+1 D$. In this regime, the most interesting perturbations are time and longitudinal components of photon fields $\delta A_{0} \equiv a_{0}$ and $\delta A_{3} \equiv a_{3}$. For the right-handed current indicated by the subscript $r$ in the background magnetic field, the Ward identity is given by

$$
\partial_{\mu} J_{r}^{\mu}=\frac{1}{(2 \pi)^{2}} E_{3} B
$$

Here, $E_{3}=\partial_{0} a_{3}-\partial_{3} a_{0}$ is an electric field induced by perturbations. With the Fourier transforming equation (4) and doing variation with respect to $a_{0}$ and $a_{3}$, we obtain the following constraints:

$$
\begin{aligned}
& q_{\mu} \Pi_{R, r}^{\mu 0}=\frac{1}{(2 \pi)^{2}}\left(-q_{3}\right) B, \\
& q_{\mu} \Pi_{R, r}^{\mu 3}=\frac{1}{(2 \pi)^{2}} q_{0} B .
\end{aligned}
$$

Note that the anomalous Ward identity (5) is satisfied by the correlator of the right-handed and vector current $\Pi_{R, r}^{\mu \nu} \equiv\left\langle J_{r}^{\mu} J^{\nu}\right\rangle_{R}$. To obtain the self-energy of the photon, the correlator of the left-handed and vector current $\Pi_{R, l}^{\mu \nu} \equiv$ $\left\langle J_{l}^{\mu} J^{\nu}\right\rangle_{R}$ needs to be added. The photon self-energy given by the sum of the right-handed and left-handed contributions is nonanomalous, as required by conservation of the vector current. With an abuse of language, we will use selfenergy to refer to $\Pi_{R, r}^{\mu \nu}$ and $\Pi_{R, l}^{\mu \nu}$ as well.

\section{CHIRAL KINETIC EQUATIONS WITH LANDAU LEVELS}

The chiral kinetic equations with Landau level states of the right-handed fermion are given by [22]

$$
\begin{aligned}
\Delta_{0} j^{0}+\Delta_{i} j^{i} & =0, \\
p_{0} j^{0}+p_{i} j^{i} & =0, \\
\Delta_{0} j^{i}+\Delta_{i} j^{0}+2 \epsilon^{i j k} p_{j} j^{k} & =0, \\
-p_{0} j^{i}-p_{i} j^{0}+\frac{1}{2} \epsilon^{i j k} \Delta_{j} j^{k} & =0,
\end{aligned}
$$

with $\Delta_{\mu}=\partial_{\mu}^{X}-\left(F_{\mu \nu}+f_{\mu \nu}\right) \frac{\partial}{\partial p_{\nu}}$ for $\mu=0,1,2,3$. We use greek letters for spacetime indices and small roman letters for spatial indices. $F_{\mu \nu}$ corresponds to the background magnetic field with the only nonvanishing components $F_{12}=-F_{21}=-B . f_{\mu \nu}=\partial_{\mu}^{X} a_{\nu}-\partial_{\nu}^{X} a_{\mu}$ corresponds to the perturbation of the electromagnetic field. Equation (6) is derived based on an expansion in $\hbar$ or, equivalently, in $\partial_{X}$ with $f_{\mu \nu} \sim O\left(\partial_{X}\right)$ but $B \sim O\left(\partial_{X}^{0}\right)$. It is valid up to $O\left(\partial_{X}\right)$ and to all order in $B$, which implicitly assumes the hierarchy of scales $\partial_{X} \ll p$. Solving (6) we can obtain $j^{\mu}$ and the momentum integral of $j^{\mu}$ gives the induced current $J^{\mu}$

$$
J^{\mu}(X)=\int d^{4} p j^{\mu}(X, p) .
$$

In the absence of perturbation, the background in the lowest Landau level (LLL) approximation is given by

$j^{0}=j^{3}=\frac{2}{(2 \pi)^{3}} \delta\left(p_{0}+p_{3}\right) e^{-p_{T}^{2} / B} f\left(\left|p_{0}\right|\right), \quad j^{1}=j^{2}=0$,

with $p_{T}^{2}=p_{1}^{2}+p_{2}^{2}$ and $f_{ \pm}\left(\left|p_{0}\right|\right)=\frac{1}{e^{\left(\left|p_{0}\right| \mp \mu_{r}\right) / T}+1}$ being the Fermi-Dirac distribution. The upper/lower sign is for positively/negatively charged LLL states. The subscript $r$ in $\mu$ indicates it is a chemical potential for right-handed 
fermions. Higher LL states are massive from the $1+1 D$ point of view with mass $\sim \sqrt{n B}$; thus, their contributions are exponentially suppressed $\sim e^{-\sqrt{n B} / T}$ in the large $B$ limit. For the LLL background, the momentum dependence factorizes into $e^{-p_{T}^{2} / B}$, which is from the LLL wave function, and $f\left(\left|p_{0}\right|\right)=f\left(\left|p_{3}\right|\right)$. The dispersion $p_{0}=-p_{3}$ is due to our nonstandard convention for momentum.

Since (6) is valid to $O\left(\partial_{X}\right)$, we seek the solution of $j^{\mu}$ order by order in the following gradient:

$$
\delta j^{\mu}=\delta j_{(0)}^{\mu}+\delta j_{(1)}^{\mu}+\cdots,
$$

with the subscript indicating the order of the gradient. We use $\delta$ to distinguish the induced $j^{\mu}$ from the background one. The terms of $O\left(\partial_{X}^{2}\right)$ are beyond the accuracy of (6). Substituting (9) into (6), we obtain to order $O\left(\partial_{X}^{0}\right)$ and $O\left(\partial_{X}\right)$, respectively,

$$
\begin{aligned}
D_{i} \delta j_{(0)}^{i} & =0, \\
D_{i} \delta j_{(0)}^{0}+2 \epsilon^{i j k} p_{j} \delta j_{(0)}^{k} & =0, \\
p_{0} \delta j_{(0)}^{0}+p_{i} \delta j_{(0)}^{i} & =0, \\
-p_{0} \delta j_{(0)}^{i}-p_{i} \delta j_{(0)}^{0}+\frac{1}{2} \epsilon^{i j k} D_{j} \delta j_{(0)}^{k} & =0,
\end{aligned}
$$

and

$$
\begin{aligned}
D_{i} \delta j_{(1)}^{i} & =S, \\
D_{i} \delta j_{(1)}^{0}+2 \epsilon^{i j k} p_{j} \delta j_{(1)}^{k} & =V_{i}^{1}, \\
p_{0} \delta j_{(1)}^{0}+p_{i} \delta j_{(1)}^{i} & =0, \\
-p_{0} \delta j_{(1)}^{i}-p_{i} \delta j_{(1)}^{0}+\frac{1}{2} \epsilon^{i j k} D_{j} \delta j_{(1)}^{k} & =V_{i}^{2},
\end{aligned}
$$

with the right-hand side defined as

$$
\begin{aligned}
S & =-\left(\delta \Delta_{0} j^{0}+\delta \Delta_{i} j^{i}+\partial_{0} \delta j_{(0)}^{0}+\partial_{i} \delta j_{(0)}^{i}\right), \\
V_{i}^{1} & =-\left(\delta \Delta_{0} j^{i}+\delta \Delta_{i} j^{0}+\partial_{0} \delta j_{(0)}^{i}+\partial_{i} \delta j_{(0)}^{0}\right), \\
V_{i}^{2} & =-\left(\frac{1}{2} \epsilon^{i j k} \delta \Delta_{j} j^{k}+\frac{1}{2} \epsilon^{i j k} \partial_{j} \delta j_{(0)}^{k}\right) .
\end{aligned}
$$

We have defined $D_{i}=-\frac{\partial}{\partial p_{j}} F_{i j}$ and $\delta \Delta_{\mu}=-\frac{\partial}{\partial p_{\nu}} f_{\mu \nu}$. We also use the shorthand notation $\partial_{\mu}=\partial_{\mu}^{X}$. The structure of the equations are quite informative: (10) and (11) can be viewed as equations for $\delta j_{(0)}^{\mu}$ and $\delta j_{(1)}^{\mu}$, respectively. The only difference is that the former are homogeneous and the latter are inhomogeneous. The homogeneous equations cannot uniquely determine $\delta j_{(0)}^{\mu}$. The source of inhomogeneous equations involves perturbations and the undetermined $\delta j_{(0)}^{\mu}$. Nevertheless, (10) and (11) can still be solved thanks to the over-determinacy of the equations.
The situation is similar to conventional CKT, where the over-determinacy of equations can be used to fix homogeneous and inhomogeneous solutions at the same order in the gradient expansion; see, for example, [64]. In CKT with Landau levels, the homogeneous solution $\delta j_{(0)}^{\mu}$ and inhomogeneous solution $\delta j_{(1)}^{\mu}$ appear at different orders.

Before closing this section, we verify that the first equation of (11) is consistent with the anomalous Ward identity. We integrate the equation over the four-momentum and reorganize it as

$$
\int d^{4} p\left(\partial_{\mu} \delta j_{(0)}^{\mu}\right)=-\int d^{4} p\left(D_{i} \delta j_{(1)}^{i}+\delta \Delta_{0} j^{0}+\delta \Delta_{3} j^{3}\right) .
$$

By our nonstandard convention, we identify the lhs as $\partial_{\mu} J^{\mu}$. For the rhs, the first term becomes the boundary term upon integration over the transverse momentum

$$
\int d^{2} p_{T} D_{i} \delta j_{(1)}^{i}=\int d^{2} p_{T} \frac{\partial}{\partial p_{M}} B \epsilon^{M N} \delta j_{(0)}^{N}=0,
$$

with the capital roman letters running over indices in the plane perpendicular to the background magnetic field $M, N=1,2$. The second terms can be written explicitly as

$$
\begin{gathered}
\int d^{4} p\left[\left(\frac{\partial}{\partial p_{M}} f_{0 M}+\frac{\partial}{\partial p_{3}} f_{03}\right) j^{0}\right. \\
\left.+\left(\frac{\partial}{\partial p_{0}} f_{30}+\frac{\partial}{\partial p_{M}} f_{3 M}\right) j^{3}\right] .
\end{gathered}
$$

The terms involving $\frac{\partial}{\partial p_{M}}$ vanish for the same reason as above. Using (8) and including contributions from both positively and negatively charged LLL states, we can combine the remaining terms as

$$
\begin{gathered}
\int d^{4} p\left(\frac{\partial}{\partial p_{3}}-\frac{\partial}{\partial p_{0}}\right) f_{03} \frac{2}{(2 \pi)^{3}} \exp \left(-p_{T}^{2} / B\right) \\
\times \delta\left(p_{0}+p_{3}\right)\left(f_{+}\left(\left|p_{0}\right|\right)-f_{-}\left(\left|p_{0}\right|\right)\right) \\
=\frac{E_{3} B}{(2 \pi)^{2}}\left(\int_{0}^{\infty} d p_{0}\left(-\frac{\partial}{\partial p_{0}}\right) f_{+}\left(\left|p_{0}\right|\right)\right. \\
\left.\quad-\int_{-\infty}^{0} d p_{0}\left(-\frac{\partial}{\partial p_{0}}\right) f_{-}\left(\left|p_{0}\right|\right)\right) \\
=\frac{E_{3} B}{(2 \pi)^{2}}\left(f_{+}(0)+f_{-}(0)\right)=\frac{E_{3} B}{(2 \pi)^{2}}
\end{gathered}
$$

Therefore, we reproduce anomalous Ward identity. There is a caveat in the above derivation in that we have taken the IR momentum cutoff $\Lambda$ to zero. Note that the validity of the kinetic theory requires $p \gg \partial_{X}$; thus, we expect $\Lambda \gtrsim \partial_{X}$. It follows that the factor $f_{+}(\Lambda)+f_{-}(\Lambda)=1+O\left(\partial_{X}\right)$. Note that $E_{3} \sim O\left(\partial_{X}\right)$ and $B \sim O\left(\partial_{X}^{0}\right)$. What (16) shows is that 
the anomalous Ward identity is satisfied up to corrections of $O\left(\partial_{X}^{2}\right)$. We will not attempt to obtain this correction as it lies beyond the accuracy of the kinetic theory. It is also interesting to note that in conventional CKT without the background magnetic field, the anomaly appears by virtue of level crossing inside the region of $|p|<\Lambda$; see, for example, [34]. In CKT with the Landau level basis, an anomaly appears manifestly from the level crossing of the LLL state alone. Below we will simply set $\Lambda=0$ in the remainder of the paper.

\section{SELF-ENERGY FROM SOLUTIONS TO KINETIC EQUATIONS}

In this section, we present solutions to (10) and (11), which allow us to extract components of self-energy in different kinematics, which in fact correspond to different states. After presenting three simple solutions, we will show that no more solutions are possible. We will argue that this is an artifact of the collisionless limit.

\section{A. Parallel $\boldsymbol{E}$ field: Chiral magnetic wave}

We begin with the case of the parallel $E$ field, which can be induced by either $a_{0}\left(t, x_{3}\right)$ or $a_{3}\left(t, x_{3}\right)$. This case can be simplified by noting that the parallel electric field only induces the longitudinal motion of LLL states, which is classical. Therefore, the motion leads only to the redistribution of LLL states like in classical kinetic theory. On the other hand, we show in [22] that the LLL state with arbitrary distribution $f\left(p_{0}\right)$ satisfies the CKT equation (6) [65]. Note that (10) is nothing but truncation of (6) to order $O\left(\partial_{X}^{0}\right)$. Thus, a solution corresponding to the redistribution of LLL states with a slow-varying $f\left(p_{0}, X\right)$ satisfies (10) automatically. Since we don't expect solution other than redistribution of LLL states, we set $\delta j_{(1)}^{\mu}=0$. In order for (11) to hold, we need to require the inhomogeneous terms to vanish: $S=V_{i}^{1}=V_{i}^{2}=0$. This allows us to fix $\delta j_{(0)}^{\mu}$ from the following constraint equations:

$$
f_{03}\left(\frac{\partial}{\partial p_{3}}-\frac{\partial}{\partial p_{0}}\right) j^{0}-\left(\partial_{0}+\partial_{3}\right) \delta j_{(0)}^{0}=0 .
$$

In arriving at (17), we have used the property of the LLL state $\delta j_{(0)}^{0}=\delta j_{(0)}^{3}$ and assumed all $\partial_{M}$ to vanish because the field perturbation $f_{03}=\partial_{0} a_{3}-\partial_{3} a_{0}$ is independent on $x_{T}$.

Equation (17) can be solved easily in momentum space as

$$
\begin{aligned}
\delta j_{(0)}^{0}=\delta j_{(0)}^{3}= & \frac{2}{(2 \pi)^{3}} \exp \left(-p_{T}^{2} / B\right) \delta\left(p_{0}+p_{3}\right) \\
& \times f^{\prime}\left(p_{0}\right) \frac{q_{3}}{q_{0}+i \epsilon+q_{3}} a_{0}, \\
\delta j_{(0)}^{0}=\delta j_{(0)}^{3}= & -\frac{2}{(2 \pi)^{3}} \exp \left(-p_{T}^{2} / B\right) \delta\left(p_{0}+p_{3}\right) \\
& \times f^{\prime}\left(p_{0}\right) \frac{q_{0}}{q_{0}+i \epsilon+q_{3}} a_{3},
\end{aligned}
$$

for perturbations $a_{0}\left(t, x_{3}\right)$ and $a_{3}\left(t, x_{3}\right)$, respectively. Again $f\left(p_{0}\right)$ can be $f_{ \pm}\left(\left|p_{0}\right|\right)$ for solutions corresponding to positively and negatively charged LLL states. We have made the substitution $q_{0} \rightarrow q_{0}+i \epsilon$ so that the solution corresponds to the retarded response.

Integrating the solution (18) over the four-momentum and using the following identity

$$
\begin{gathered}
\int_{0}^{\infty} d p_{0} f_{+}^{\prime}\left(\left|p_{0}\right|\right)-\int_{-\infty}^{0} d p_{0} f_{-}^{\prime}\left(\left|p_{0}\right|\right) \\
=-f_{+}(0)-f_{-}(0)=-1,
\end{gathered}
$$

we obtain the following retarded self-energy components from (3):

$$
\begin{aligned}
& \Pi_{R, r}^{00}\left(q_{0}, q_{3}\right)=\Pi_{R, r}^{30}\left(q_{0}, q_{3}\right)=-\frac{e^{3} B}{(2 \pi)^{2}} \frac{q_{3}}{q_{0}+i \epsilon+q_{3}}, \\
& \Pi_{R, r}^{03}\left(q_{0}, q_{3}\right)=\Pi_{R, r}^{33}\left(q_{0}, q_{3}\right)=\frac{e^{3} B}{(2 \pi)^{2}} \frac{q_{0}}{q_{0}+i \epsilon+q_{3}} .
\end{aligned}
$$

We have reinstated the powers of $e$ in the above. Note that (20) is independent of temperature and chemical potential. This is because the integration over $p_{0}$ only picks up boundary terms at $p_{0}=0$, similar to (16). We can verify (20) indeed satisfies (5). The pole of $\Pi_{R, r}^{\mu \nu}$ gives the dispersion relation $q_{0}+q_{3}=0$ of the collective excitation of the chiral medium. It corresponds to a wave propagating with the speed of light in the $x_{3}$ direction. In fact, this is nothing but the chiral magnetic wave in the limit of the strong magnetic field [15].

It is also interesting to note that $\Pi_{R, r}^{\mu \nu}$ is not symmetric with respect to Lorentz indices. The reason is that the states consisting of right-handed fermions are not parity invariant. Applying parity transformation on (20), we obtain the components of self-energy for the medium consisting of left-handed fermions

$$
\begin{aligned}
\Pi_{R, l}^{00}\left(q_{0}, q_{3}\right) & =-\Pi_{R, l}^{30}\left(q_{0}, q_{3}\right)=\frac{e^{3} B}{(2 \pi)^{2}} \frac{q_{3}}{q_{0}+i \epsilon-q_{3}}, \\
-\Pi_{R, l}^{03}\left(q_{0}, q_{3}\right) & =\Pi_{R, l}^{33}\left(q_{0}, q_{3}\right)=\frac{e^{3} B}{(2 \pi)^{2}} \frac{q_{0}}{q_{0}+i \epsilon-q_{3}} .
\end{aligned}
$$

These results can also be obtained from explicit solutions of the chiral kinetic theory for the left-handed medium, which we collect in Appendix A. Adding up contributions from both left-handed and right handed fermions, we obtain the following components of self-energy for the parity invariant state: 


$$
\begin{aligned}
& \Pi_{R}^{00}\left(q_{0}, q_{3}\right)=\frac{e^{3} B}{(2 \pi)^{2}} \frac{2 q_{3}^{2}}{\left(q_{0}+i \epsilon\right)^{2}-q_{3}^{2}}, \\
& \Pi_{R}^{03}\left(q_{0}, q_{3}\right)=\Pi_{R}^{30}\left(q_{0}, q_{3}\right)=-\frac{e^{3} B}{(2 \pi)^{2}} \frac{2 q_{0} q_{3}}{\left(q_{0}+i \epsilon\right)^{2}-q_{3}^{2}}, \\
& \Pi_{R}^{33}\left(q_{0}, q_{3}\right)=\frac{e^{3} B}{(2 \pi)^{2}} \frac{2 q_{0}^{2}}{\left(q_{0}+i \epsilon\right)^{2}-q_{3}^{2}} .
\end{aligned}
$$

These agree with field theoretic results in the LLL approximation up to an overall factor of $e^{-q_{T}^{2} / B}$ [66]. The exponential factor is at least $O\left(\partial_{X}^{2}\right)$, which lies beyond the accuracy of our current chiral kinetic equation.

\section{B. Static perpendicular $\boldsymbol{E}$ field: Drift state}

Next we consider the case of static perpendicular $E$ induced by $a_{0}\left(x_{T}\right)$. We begin by solving for $\delta j_{(1)}^{0}$ and $\delta j_{(1)}^{3}$ from the third and $i=3$ component of the fourth equations of (11) to obtain

$$
\left(\begin{array}{c}
\delta j_{(1)}^{0} \\
\delta j_{(1)}^{3}
\end{array}\right)=\frac{1}{p_{0}^{2}-p_{3}^{2}}\left(\begin{array}{c}
-p_{0} p_{M} \delta j_{(1)}^{M}-p_{3} \frac{1}{2} \epsilon^{M N} D_{M} \delta j_{(1)}^{N} \\
p_{3} p_{M} \delta j_{(1)}^{M}+p_{0} \frac{1}{2} \epsilon^{M N} D_{M} \delta j_{(1)}^{N}
\end{array}\right) .
$$

We proceed with the following ansatz:

$$
\delta j_{(1)}^{M} \propto \exp \left(-p_{T}^{2} / B\right) \delta\left(p_{0}+p_{3}\right),
$$

which converts (11) to the following equivalent equations: $\frac{B \delta j_{(1)}^{M}}{2\left(p_{0}+p_{3}\right)}-p_{0} \delta j_{(1)}^{M}=-\frac{1}{2} \epsilon^{M N} E_{N} \frac{\partial}{\partial p_{0}} j^{0}-\frac{1}{2} \epsilon^{M N} \partial_{N} \delta j_{(0)}^{3}$, $2 \epsilon^{M N} p_{M} \delta j_{(1)}^{N}=-\frac{2 E_{M} p_{M}}{B} j^{0}$,

$$
\frac{\epsilon^{M N} \delta j_{(1)}^{N} B}{p_{0}+p_{3}}+2 \epsilon^{M N} \delta j_{(1)}^{N} p_{3}=E_{M} \frac{\partial}{\partial p_{0}} j^{0}+\partial_{M} \delta j_{(0)}^{0},
$$

with $E_{N}=-\partial_{N} a_{0}$ being the perpendicular electric field perturbation. Equation (25) adopts the following solution:

$$
\begin{aligned}
\delta j_{(0)}^{0}= & \delta j_{(0)}^{3}=a_{0}\left(-\delta^{\prime}\left(p_{0}+p_{3}\right)-\frac{2 p_{0}}{B} \delta\left(p_{0}+p_{3}\right)\right) \\
& \times \exp \left(-p_{T}^{2} / B\right) f\left(p_{0}\right), \\
\delta j_{(1)}^{M}= & \frac{\epsilon^{M N} E_{N}}{B} \delta\left(p_{0}+p_{3}\right) \exp \left(-p_{T}^{2} / B\right) f\left(p_{0}\right), \\
\delta j_{(1)}^{0}= & \delta j_{(1)}^{3}=\frac{\epsilon^{M N} p_{M} E_{N}}{B} \delta^{\prime}\left(p_{0}+p_{3}\right) \exp \left(-p_{T}^{2} / B\right) f\left(p_{0}\right) .
\end{aligned}
$$

Using $\delta^{\prime}\left(p_{0}+p_{3}\right) f\left(p_{0}\right)=-\delta\left(p_{0}+p_{3}\right) f^{\prime}\left(p_{0}\right)$, we see that the zeroth order solution $\delta j_{(0)}^{\mu}$ is simply a redistribution of
LLL states with $\delta f\left(p_{0}\right)=a_{0}\left(f^{\prime}\left(p_{0}\right)-\frac{2 p_{0}}{B} f\left(p_{0}\right)\right)$; thus, (10) is automatically satisfied.

Integrating the solution over the four-momentum and using the following integrals:

$$
\begin{aligned}
& \int_{0}^{\infty} d p_{0} p_{0} f_{+}\left(\left|p_{0}\right|\right)-\int_{-\infty}^{0} d p_{0} p_{0} f_{-}\left(\left|p_{0}\right|\right)=\frac{\pi^{2} T^{2}}{6}+\frac{\mu_{r}^{2}}{2}, \\
& \int_{0}^{\infty} d p_{0} f_{+}\left(\left|p_{0}\right|\right)-\int_{-\infty}^{0} d p_{0} f_{-}\left(\left|p_{0}\right|\right)=\mu_{r},
\end{aligned}
$$

we find the following components of self-energy after reinstating powers of $e$ :

$$
\begin{aligned}
& \Pi_{R, r}^{00}\left(q_{M}\right)=\Pi_{R, r}^{30}\left(q_{M}\right)=-\frac{e^{2}}{(2 \pi)^{2}}\left(e B+\frac{\pi^{2} T^{2}}{3}+\mu_{r}^{2}\right), \\
& \Pi_{R, r}^{M 0}\left(q_{M}\right)=-\frac{e^{2}}{(2 \pi)^{2}} i \epsilon^{M N} q_{N} \mu_{r} .
\end{aligned}
$$

Equation (27) satisfies (5) trivially because $q_{0}=q_{3}=0$. Note that $\delta j_{(1)}^{0}$ and $\delta j_{(1)}^{3}$ are the odd function of $p_{T}$, which vanishes upon integration over $p_{T}$, and thus do not contribute to self-energy. A counterpart for the left-handed contribution can be obtained by parity transformation as in the previous case. Note that under parity transformation $\mu_{r} \rightarrow \mu_{l}$, we arrive at

$$
\begin{aligned}
\Pi_{R, l}^{00}\left(q_{M}\right) & =-\Pi_{R, l}^{30}\left(q_{M}\right)=-\frac{e^{2}}{(2 \pi)^{2}}\left(e B+\frac{\pi^{2} T^{2}}{3}+\mu_{l}^{2}\right), \\
-\Pi_{R, l}^{M 0}\left(q_{M}\right) & =\frac{e^{2}}{(2 \pi)^{2}} i \epsilon^{M N} q_{N} \mu_{l} .
\end{aligned}
$$

Adding up contributions from both (27) and (28), we obtain the following nonvanishing components for photon selfenergy:

$$
\begin{aligned}
\Pi_{R}^{00}\left(q_{M}\right) & =-\frac{2 e^{2}}{(2 \pi)^{2}}\left(e B+\frac{\pi^{2} T^{2}}{3}+\mu^{2}+\mu_{5}^{2}\right), \\
\Pi_{R}^{30}\left(q_{M}\right) & =-\frac{4 e^{2}}{(2 \pi)^{2}} \mu \mu_{5} \\
\Pi_{R}^{M 0}\left(q_{M}\right) & =-\frac{2 e^{2}}{(2 \pi)^{2}} i \epsilon^{M N} q_{N} \mu
\end{aligned}
$$

with $\mu=\left(\mu_{r}+\mu_{l}\right) / 2$ and $\mu_{5}=\left(\mu_{r}-\mu_{l}\right) / 2$. Note that unlike the case of the parallel electric field, the case of the perpendicular electric field gives rise to medium dependent self-energy components. In particular, the medium dependent terms in $\Pi_{R}^{00}$ and $\Pi_{R}^{30}$ would not appear in the static limit $q_{0} \rightarrow 0$ of (22). The difference can be understood as follows: the solutions from parallel and perpendicular electric fields correspond to a different state: the parallel electric field leads to the redistribution of LLL states, while the perpendicular electric field leads to a drift 
state, with the medium drifting with a velocity orthogonal to both $E_{M}$ and $B$. In the drift state, Hall current is expected and is consistent with $\Pi_{R}^{M 0}$ above. $\Pi_{R}^{00}$ and $\Pi_{R}^{30}$ give the deviation of charge density and current density of the drift state from those of the background. Interestingly, the deviation coincides with the 00 component of the selfenergy in the chiral medium without the background magnetic field [67].

We can gain further insight into the drift state by "boosting" the equilibrium state. It is not difficult to see that by boosting the equilibrium medium to a velocity $-\frac{\epsilon^{M N} E_{N}}{B}$, we will then have the orthogonal electric field $E_{M}$ and background magnetic field $B$ [68]. To describe it more quantitatively, we use the covariant form of the background solution

$j^{\mu}=\frac{2}{(2 \pi)^{3}} \delta(p \cdot(u+b)) e^{-p_{T}^{2} / B} f(p \cdot u)(u+b)^{\mu}$,

which generalizes the solution in the medium frame to arbitrary frame. We verify in Appendix B that it indeed satisfies the covariant chiral kinetic equations to the lowest order in gradient. Here $u^{\mu}$ and $b^{\mu}$ denote fluid velocity and magnetic direction and $p_{T}^{2}=-p^{2}+(p \cdot u)^{2}-(p \cdot b)^{2} \cdot u^{\mu}$ and $b^{\mu}$ are orthogonal to each other $u \cdot b=0$. In the medium frame, we have $u^{\mu}=(1,0,0,0)$ and $b^{\mu}=$ $(0,0,0,1)$. Under the boost, we have $\delta u^{M}=\frac{\epsilon^{M N} E_{N}}{B} \sim$ $O\left(\partial_{X}\right)$ and $\delta b=0$. It is easy to see that $\delta u^{M}$ leads to $\delta j_{(1)}^{M}$. The remaining corrections are not from covariance and are only present in $\delta j^{0}$ and $\delta j^{3}$. At zeroth order $\delta j_{(0)}^{0}$ and $\delta j_{(0)}^{3}$ come from the fact that the electric field perturbation is not constant but $x_{T}$ dependent. In fact, $a_{0}$ in (26) should be interpreted as $a_{0} \sim E_{M} / \partial_{M}$; thus, the zeroth order correction characterizes the redistribution of LL states in response to perturbation. A similar correction to the zeroth order solution is also present in chiral kinetic theory without the background field [33]. At the first order, $\delta j_{(1)}^{0}$ and $\delta j_{(1)}^{3}$ are entirely determined by $\delta j_{(1)}^{M}$ from (23), which as we discussed above is not sensitive to the $x_{T}$ dependence of the perturbation. Note that $\delta^{\prime}\left(p_{0}+p_{3}\right) f\left(p_{0}\right)=-\delta\left(p_{0}+p_{3}\right) f^{\prime}\left(p_{0}\right)$. It is suggestive to interpret $\delta j_{(1)}^{0}$ and $\delta j_{(1)}^{3}$ as a modification of the distribution function $f\left(p_{0}\right) \rightarrow f\left(p_{0}\right)-\frac{\epsilon^{M N} p_{M} E_{N}}{B} f^{\prime}\left(p_{0}\right)=$ $f\left(p_{0}-\frac{\epsilon^{M N} p_{M} E_{N}}{B}\right)$, or $p_{0} \rightarrow p_{0}-\frac{\epsilon^{M N} p_{M} E_{N}}{B}$. This is analogous to the side-jump effect in the momentum in the absence of a background field $[38,69]$. Note that since our background solution is homogeneous in coordinate, a possible jump in coordinate is not visible from our comparison. We should not confuse the frame vector frequently used in the description of the side jump with the fluid velocity $u^{\mu}$. The latter is needed to define the magnetic field in the background.

\section{Static perpendicular $B$ field: Tilted state}

We turn to the case of the static perpendicular magnetic field induced by $a_{3}\left(x_{T}\right)$. The analysis is similar to the previous subsection. We will not spell out the details. With the ansatz $\delta j_{(1)}^{M} \propto \exp \left(-p_{T}^{2} / B\right) \delta\left(p_{0}+p_{3}\right)$, we obtain the following solution:

$\delta j_{(0)}^{0}=\delta j_{(0)}^{3}=\left(-\frac{2 p_{0}}{B} \delta\left(p_{0}+p_{3}\right) \exp \left(-p_{T}^{2} / B\right) f\left(p_{0}\right)\right) a_{3}$,

$\delta j_{(1)}^{0}=\delta j_{(1)}^{3}=\frac{p_{M} B_{M}^{\perp}}{B} \delta^{\prime}\left(p_{0}+p_{3}\right) \exp \left(-p_{T}^{2} / B\right) f\left(p_{0}\right)$,

$\delta j_{(1)}^{M}=\frac{B_{M}^{\perp}}{B} \delta\left(p_{0}+p_{3}\right) \exp \left(-p_{T}^{2} / B\right) f\left(p_{0}\right)$,

with $B_{M}^{\perp}=-\epsilon^{M N} \partial_{N} a_{3}$ being the perpendicular magnetic field. It gives rise to the following components of selfenergy:

$$
\begin{aligned}
& \Pi_{R, r}^{03}\left(q_{M}\right)=\Pi_{R, r}^{33}\left(q_{M}\right)=-\frac{e^{2}}{(2 \pi)^{2}}\left(\frac{\pi^{2} T^{2}}{3}+\mu_{r}^{2}\right), \\
& \Pi_{R, r}^{M 3}\left(q_{M}\right)=-\frac{e^{2}}{(2 \pi)^{2}} i \epsilon^{M N} q_{N} \mu_{r},
\end{aligned}
$$

which again satisfies (5) trivially as $q_{0}=q_{3}=0$. The contribution from left-handed fermions can be obtained by the parity transformation to give

$$
\begin{aligned}
-\Pi_{R, l}^{03}\left(q_{M}\right) & =\Pi_{R, l}^{33}\left(q_{M}\right)=-\frac{e^{2}}{(2 \pi)^{2}}\left(\frac{\pi^{2} T^{2}}{3}+\mu_{l}^{2}\right), \\
\Pi_{R, l}^{M 3}\left(q_{M}\right) & =\frac{e^{2}}{(2 \pi)^{2}} i \epsilon^{M N} q_{N} \mu_{l} .
\end{aligned}
$$

The combined photon self-energy components are

$$
\begin{aligned}
\Pi_{R}^{03} & =-\frac{4 e^{2}}{(2 \pi)^{2}} \mu \mu_{5}, \\
\Pi_{R}^{33}\left(q_{M}\right) & =-\frac{2 e^{2}}{(2 \pi)^{2}}\left(\frac{\pi^{2} T^{2}}{3}+\mu^{2}+\mu_{5}^{2}\right), \\
\Pi_{R}^{M 3}\left(q_{M}\right) & =-\frac{2 e^{2}}{(2 \pi)^{2}} i \epsilon^{M N} q_{N} \mu_{5} .
\end{aligned}
$$

Comparing (32) with the static limit of 03 and 33 components of (22), which vanishes identically, we see the difference is also medium dependent. We can see that $\Pi_{R}^{M 3}$ gives precisely the chiral magnetic effect due to $B_{\perp}$ :

$J^{M}=\Pi_{R}^{M 3} a_{3}=-\frac{2 e^{2}}{(2 \pi)^{2}} \epsilon^{M N} \partial_{N} a_{3} \mu_{5}=\frac{2 e^{2}}{(2 \pi)^{2}} \mu_{5} B_{M}^{\perp}$.

$\Pi_{R}^{M 3}$ also agrees with components of parity odd self-energy in the absence of the background field [33], which is 
responsible for the chiral magnetic effect. These particular components of self-energy actually give rise to chiral plasma instability [67]. We can see some trace from the backreaction of the induced chiral magnetic current to the electromagnetic field. The induced magnetic field $\Delta B$ can be found by solving the Maxwell equation

$$
(\nabla \times \Delta B)^{M}=J^{M},
$$

with the solution given by $\Delta B_{i}=\frac{2 e^{2}}{(2 \pi)^{2}} \mu_{5} a_{3} \delta_{i 3}$. It can lead to the enhancement of the background magnetic field. However, to answer the question dynamically, we need to know the self-energy beyond the static limit.

Let us again compare the tilted state with the equilibrium state rotated in such a way that the background magnetic field coincides with that of the tilted state [70]. We can use the covariant form of the background solution (30) with $\delta b^{M}=B_{M}^{\perp} / B$ and $\delta u=0 . \delta b^{M}$ gives precisely $\delta j_{(1)}^{M}$ from the covariant factor $(u+b)^{\mu}$. The remaining difference between the tilted state and equilibrium state is in $\delta j^{0}$ and $\delta j^{3}$. The tilted state is not entirely equivalent to the rotated equilibrium state because of the $x_{T}$ dependence of the perpendicular magnetic field. The $x_{T}$ dependence leads to the difference in $\delta j_{(0)}^{0}$ and $\delta j_{(0)}^{3}$. On the contrary, $\delta j_{(1)}^{0}$ and $\delta j_{(1)}^{3}$ are fixed by $\delta j_{(1)}^{M}$ through (23); thus, they are not sensitive to the $x_{T}$ dependence of the perturbation. Using $\delta^{\prime}\left(p_{0}+p_{3}\right) f\left(p_{0}\right)=-\delta\left(p_{0}+p_{3}\right) f^{\prime}\left(p_{0}\right)$, we interpret $\delta j_{(1)}^{0}$ and $\delta j_{(1)}^{3}$ as modifications of the distribution function: $f\left(p_{0}\right) \rightarrow f\left(p_{0}\right)-\frac{p_{M} B_{M}}{B} f^{\prime}\left(p_{0}\right)=f\left(p_{0}-\frac{p_{M} B_{M}}{B}\right)$, or $p_{0} \rightarrow p_{0}-\frac{p_{M} B_{M}}{B}$. Like in the case of the drift state, it is suggestive to interpret the modification of distribution function as an analog to the side-jump effect in the momentum in the absence of the background field $[38,69]$. Since our background solution is homogeneous in the coordinate, a possible jump in coordinate is not visible. We stress that this is a new effect due to the background field: the conventional side jump is manifested through the boost, and in our case the side jump can be manifested through both boost $\delta u$ and rotation $\delta b$, as we see in both the drift state and tilted state, respectively.

\section{No more solutions}

Finally, we look for solutions for more general perturbations without using the ansatz. Since the background is LLL states in equilibrium and the zeroth order solution $\delta j_{(0)}^{\mu}$ is a redistribution of LLL [71], we have $j_{0}=j_{3}$ and $\delta j_{(0)}^{0}=\delta j_{(0)}^{3}$ with all other components vanishing. It follows that $S=V_{3}^{1}$ and $\frac{1}{2} \epsilon^{M N} V_{N}^{1}=V_{M}^{2}$. We can eliminate redundant equations in (11) to obtain

$$
\begin{aligned}
& D_{M} \delta j_{(1)}^{M}=2 \epsilon^{M N} p_{M} \delta j_{(1)}^{N}, \\
& \left(\frac{1}{2} \epsilon^{M N} D_{N}+p_{M}\right)\left(\delta j_{(1)}^{0}-\delta j_{(1)}^{3}\right)+\left(p_{0}+p_{3}\right) \delta j_{(1)}^{M}=0 \\
& 2 \epsilon^{M N} p_{M} \delta j_{(1)}^{N}=-\left(\left(\delta \Delta_{0}+\delta \Delta_{3}\right) j^{0}+\left(\partial_{0}+\partial_{3}\right) \delta j_{(0)}^{0}\right) \\
& \left(p_{0}-p_{3}\right) \epsilon^{M N} \delta j_{(1)}^{N}+\left(\frac{1}{2} D_{M}+\epsilon^{M N} p_{N}\right)\left(\delta j_{(1)}^{0}+\delta j_{(1)}^{3}\right) \\
& \quad=-\left(\delta \Delta_{M} j^{0}+\partial_{M} \delta j_{(0)}^{0}\right) .
\end{aligned}
$$

To proceed, we define $A_{M}$ by pulling out a factor of $e^{-p_{T}^{2} / B}$ from $\delta j_{(1)}^{M}$ :

$$
\delta j_{(1)}^{M}=e^{-p_{T}^{2} / B} A_{M}
$$

$A^{M}$ are functions of $p$ and $q$. The solutions for static perpendicular electric and magnetic fields correspond to $A_{M}$ being independent of $p_{M}$. Plugging (38) into (37) and dividing out the common factor $e^{-p_{T}^{2} / B}$, we obtain

$$
\epsilon^{M N} \frac{\partial}{\partial p_{N}} A_{M}=0
$$

$$
-\frac{1}{p_{0}-p_{3}}\left(2 p_{M}-\frac{B}{2} \frac{\partial}{\partial p_{M}}\right) \frac{B}{2} \frac{\partial}{\partial p_{K}} A_{K}+\left(p_{0}+p_{3}\right) A_{M}=0,
$$

$$
\begin{aligned}
& 2 \epsilon^{M N} p_{M} A_{N}=-\left(\delta \Delta_{0}+\delta \Delta_{3}\right) \delta\left(p_{0}+p_{3}\right) f\left(p_{0}\right) \\
&-\delta\left(p_{0}+p_{3}\right)\left(\partial_{0}+\partial_{3}\right) g\left(p_{0}, X\right), \quad \text { (39c) } \\
&\left(p_{0}-p_{3}\right) \epsilon^{M N} A_{N}+\frac{-1}{p_{0}+p_{3}} \frac{B}{2} \epsilon^{M N} \frac{\partial}{\partial p_{N}}\left[\left(2 p_{K}-\frac{B}{2} \frac{\partial}{\partial p_{K}}\right) A_{K}\right] \\
&=-\delta \Delta_{M} \delta\left(p_{0}+p_{3}\right) f\left(p_{0}\right)-\delta\left(p_{0}+p_{3}\right) \partial_{M} g\left(p_{0}, X\right) . \quad(39 \mathrm{~d})
\end{aligned}
$$

We have defined $\delta j_{(0)}^{0}=\delta\left(p_{0}+p_{3}\right) e^{-p_{T}^{2} / B} g\left(p_{0}, X\right)$. We already know $\delta j_{(0)}^{\mu}$ corresponds to the redistribution of LLL states, so $g$ has to be a function of $p_{0}$ and $X$ only. Below we will show that this is not possible except for the special cases presented in the above subsections. We first apply $\epsilon^{M N} \frac{\partial}{\partial p_{N}}$ to (39b) and use (39a) to arrive at

$$
p_{M} \epsilon^{M N} \frac{\partial}{\partial p_{N}} \frac{\partial}{\partial p_{K}} A_{K}=0
$$

We can also apply $\epsilon^{M N} p_{N}$ to (39b) and use (40) to obtain

$$
\left(p_{0}+p_{3}\right) \epsilon^{M N} p_{M} A_{N}=0 .
$$

We then multiply (39d) by $p_{M}$ to obtain 


$$
\begin{aligned}
\left(p_{0}-\right. & \left.p_{3}\right) p_{M} \epsilon^{M N} A_{N}+\frac{-1}{p_{0}+p_{3}} \frac{B}{2} \epsilon^{M N} p_{M} \\
& \times \frac{\partial}{\partial p_{N}}\left(2 p_{K} A_{K}-\frac{B}{2} \frac{\partial}{\partial p_{K}} A_{K}\right) \\
= & -p_{M} \delta \Delta_{M} \delta\left(p_{0}+p_{3}\right) f\left(p_{0}\right) \\
& -\delta\left(p_{0}+p_{3}\right) p_{M} \partial_{M} g\left(p_{0}, X\right) .
\end{aligned}
$$

Using (41) to simplify the first term and using (40) to eliminate the second term in the round bracket on the lhs, we arrive at

$$
\begin{array}{r}
2 p_{0} \epsilon^{M N} p_{M} A_{N}+\frac{-B}{p_{0}+p_{3}}\left[\epsilon^{M N} p_{M} A_{N}+\epsilon^{M N} p_{M} p_{K} \frac{\partial}{\partial p_{N}} A_{K}\right] \\
=-p_{M} \delta \Delta_{M} \delta\left(p_{0}+p_{3}\right) f\left(p_{0}\right)-\delta\left(p_{0}+p_{3}\right) p_{M} \partial_{M} g\left(p_{0}, X\right) .
\end{array}
$$

The second term in the square bracket can be further simplified using the following identity:

$$
\epsilon^{M N} p_{K}+\epsilon^{N K} p_{M}+\epsilon^{K M} p_{N}=0 .
$$

It follows that

$$
\begin{aligned}
\epsilon^{M N} p_{M} p_{K} \frac{\partial}{\partial p_{N}} A_{K} & =-\left(\epsilon^{N K} p_{M}+\epsilon^{K M} p_{N}\right) p_{M} \frac{\partial}{\partial p_{N}} A_{K} \\
& =\epsilon^{M N} p_{M} p_{K} \frac{\partial}{\partial p_{K}} A_{N},
\end{aligned}
$$

where we have used (39a) and relabeled indices in the second equality. We can then rewrite the square bracket of (43) as

$$
\begin{aligned}
& {\left[\epsilon^{M N} p_{M} A_{N}+\epsilon^{M N} p_{M} p_{K} \frac{\partial}{\partial p_{N}} A_{K}\right]} \\
& \quad=\epsilon^{M N} p_{M}\left(1+\frac{\partial}{\partial p_{K}}\right) A_{N}=\epsilon^{M N} \frac{\partial}{\partial p_{K}} p_{M} A_{N} .
\end{aligned}
$$

With this, we arrive at the following simple form of (43):

$$
\begin{aligned}
\left(2 p_{0}\right. & \left.+\frac{-B}{p_{0}+p_{3}}\right) \epsilon^{M N} p_{M} A_{N} \\
= & -p_{M} \delta \Delta_{M} \delta\left(p_{0}+p_{3}\right) f\left(p_{0}\right) \\
& -\delta\left(p_{0}+p_{3}\right) p_{M} \partial_{M} g\left(p_{0}, X\right) .
\end{aligned}
$$

We can now plug (39c) into the above and compare the coefficients of $\delta\left(p_{0}+p_{3}\right)$ and $\delta^{\prime}\left(p_{0}+p_{3}\right)$ to determine $g\left(p_{0}\right)$. Note that the coefficients have to be matched separately rather than using $\delta^{\prime}\left(p_{0}+p_{3}\right)=-\frac{\delta\left(p_{0}+p_{3}\right)}{p_{0}+p_{3}}$, which involves the dropping of boundary terms and is not always justified. We end up with two expressions for $g$ :

$$
\begin{aligned}
g & =\frac{\tilde{f}_{30} f^{\prime}\left(p_{0}\right)}{i\left(q_{0}+q_{3}\right)}, \\
g & =\frac{\left(2 p_{0} \tilde{f}_{30}-2 p_{M} \tilde{f}_{M 0}\right) f^{\prime}\left(p_{0}\right)-\frac{2 p_{0}}{B}\left(2 p_{M} \tilde{f}_{0 M}+2 p_{M} \tilde{f}_{3 M}\right) f\left(p_{0}\right)}{2 i\left(p_{0}\left(q_{0}+q_{3}\right)-p_{M} q_{M}\right)},
\end{aligned}
$$

with $\tilde{f}_{\mu \nu}=i\left(q_{\mu} a_{\nu}-q_{\nu} a_{\mu}\right)$. It is easy to verify that (48) includes all three cases we discussed above: in the case of the parallel electric field, two expressions of (48) give the same result; in the case of the static perpendicular electric or magnetic field, only the second expression should be used. In all three cases, $g$ is independent of $p_{T}$. This is a necessary condition for $\delta j_{(0)}^{0}$ to be a valid zeroth order solution as stressed above. However, any other field perturbations would not allow for a $p_{T}$ independent $g$; thus, no more solutions can be found.

The lack of a nontrivial solution may sound odd. Indeed, it is actually an artifact of the collisionless limit we work in. In the absence of interaction between LL states, the dynamics of LL states is restricted to classical longitudinal motion. This can be induced by the parallel electric field leading to the chiral magnetic wave. The perpendicular electric or magnetic fields necessarily lead to the quantum transition between LL states. Although our background and the zeroth order solutions are restricted to be LLL states in equilibrium and its redistribution, the medium dependent contribution to self-energy components in (29) and (34) effectively go beyond LLL approximation. This is can be seen by noting the field theoretic results of the self-energy in LLL approximation is always proportional to $B$ from the density of states of LLL, but the medium dependent contributions are $O\left(B^{0}\right)$, which is likely due to higher 
LL contributions. As remarked above, the quantum transition from LLL to higher LL is not possible without interaction. The only possible solutions are static ones in which no dynamics is involved. In other words, although these static solutions can be found, solutions corresponding the dynamical realization of these states are not possible in the absence of collision. The collisionless limit also lies behind the disagreement of the static limit of (22) and (29). We expect a consistent limit will be reached by including collision. We leave it for future work.

\section{SUMMARY AND OUTLOOK}

By using chiral kinetic theory with Landau level states, we studied the photon self-energy in magnetized chiral plasma from the response to electromagnetic field perturbations. In the regime of the strong magnetic field, we studied the response of chiral plasma to three different field perturbations: the parallel electric field, and the static perpendicular electric and static perpendicular magnetic fields. They give rise to components of self-energy in different kinematics. The three perturbations lead to the chiral magnetic wave, drift state, and tilted state, respectively. From the case of the chiral magnetic wave, we obtain self-energy components, which are in agreement with field theoretic results up to the accuracy of the chiral kinetic theory. From the cases of drift state and tilted state, we obtain components of self-energy in the static case. We also compared the solutions of the drift state and tilted state with boosted and rotated background solutions, respectively. The difference is understood from the spatial dependence of the perturbations.

We further showed no solution can be found in response to other more general perturbations. We argued that it is an artifact of the collisionless limit in which we work. Without collisions, quantum transition between LL states is not possible but only the classical motion of the LL state is allowed. As a result, the drift state and tilted state cannot be realized dynamically but can only be found as static solutions. To study more general perturbations, it is crucial to introduce collision. It can be done by promoting the photon as a dynamical field, which mediates the interaction between LL states. It would also allow us to study the selfenergy of LL states and the photon in a systematic way. We hope to address these in the future.

\section{ACKNOWLEDGMENTS}

S. L. is grateful to Koichi Hattori, Defu Hou, Igor Shovkovy, and Di-Lun Yang for useful discussions. He also thanks the Yukawa Institute of Theoretical Physics for hospitality and the workshop "Quantum kinetic theories in magnetic and vortical fields" for providing a stimulating environment during the final stage of this work. This work is in part supported by NSFC under Grants No. 11675274 and No. 11735007.

\section{APPENDIX A: CHIRAL KINETIC EQUATIONS FOR LEFT-HANDED FERMION}

The chiral kinetic equation for left-handed fermions can be derived from the equation of motion for the corresponding Wigner function $W$ :

$$
\left(\frac{1}{2} \Delta_{\mu}-i p_{\mu}\right) \bar{\sigma}^{\mu} W=0, \quad\left(\frac{1}{2} \Delta_{\mu}+i p_{\mu}\right) W \bar{\sigma}^{\mu}=0 .
$$

The difference with the counterpart of right-handed fermions is $\sigma^{\mu} \rightarrow \bar{\sigma}^{\mu}$. We then decompose the Wigner function into components $j^{\mu}$ as

$$
W=j^{0} 1+j^{i} \sigma_{i}
$$

This decomposition keeps the integral representation of current (7) the same for left-handed fermions. The chiral kinetic equations for components follow immediately from (A1)

$$
\begin{aligned}
\Delta_{0} j^{0}-\Delta_{i} j^{i} & =0, \\
p_{0} j^{0}-p_{i} j^{i} & =0, \\
\Delta_{0} j^{i}-\Delta_{i} j^{0}-2 \epsilon^{i j k} p_{j} j^{k} & \\
-p_{0} j^{i}+p_{i} j^{0}-\frac{1}{2} \epsilon^{i j k} \Delta_{j} j^{k} & =0 .
\end{aligned}
$$

They are obtainable from the counterpart of right-handed fermions by the replacement $\Delta_{i} \rightarrow-\Delta_{i}$ and $p_{i} \rightarrow-p_{i}$. It follows that the contributions of left-handed fermions and right-handed fermions to self-energy are related by the replacement $q_{i} \rightarrow-q_{i}$, which agrees with what we used in the text.

\section{APPENDIX B: COVARIANCE OF THE BACKGROUND SOLUTION}

In this appendix, we show the covariance of the background solution (30). We first write down the covariant chiral kinetic equations

$$
\begin{gathered}
\Delta_{\mu} j^{\mu}=0, \\
-\Delta_{\mu} j_{\nu}+\Delta_{\nu} j_{\mu}+2 \epsilon_{\mu \nu \rho \sigma} p^{\rho} j^{\sigma}=0, \\
p_{\mu} j^{\mu}=0, \\
p_{\mu} j_{\nu}-p_{\nu} j_{\mu}+\frac{1}{2} \epsilon_{\mu \nu \rho \sigma} \Delta^{\rho} j^{\sigma}=0,
\end{gathered}
$$

with $\Delta_{\mu}=\partial_{\mu}-\frac{\partial}{\partial p_{\nu}}\left(F_{\mu \nu}+f_{\mu \nu}\right) \cdot F_{\mu \nu}$ can be decomposed as $F_{\mu \nu}=\epsilon_{\mu \nu \rho \sigma} B^{\rho} u^{\sigma}+E_{\mu} u_{\nu}-E_{\nu} u_{\mu}$. Here $u^{\mu}$ is a unit vector corresponding to fluid velocity. $E_{\mu}=F_{\mu \nu} u^{\nu}$ and $B^{\mu}=$ $\frac{1}{2} \epsilon^{\mu \nu \alpha \beta} u_{\nu} F_{\alpha \beta}$ are electric and magnetic fieldS in the fluid. We further define $B^{\mu}=B b^{\mu}$ with $b^{\mu}$ being a direction 
vector of the magnetic field. By definition we have $u \cdot b=0$. The solution (8) corresponds to $u^{\mu}=(1,0,0,0)$ and $b^{\mu}=(0,0,0,1)$. We will not consider a general $u^{\mu}$, but restrict ourselves to the case with flow $u^{\mu}=$ $(1,0,0,0)+O\left(\partial_{X}\right)$, i.e., flows which deviate from the static flow only by the gradient term. Thus $E_{\mu} \sim O\left(\partial_{X}\right)$, allowing us to neglect the electric field terms in $\Delta_{\mu}$. To the lowest order in gradient $\Delta_{\mu}=\frac{\partial}{\partial p_{\nu}} B \epsilon_{\mu \nu \rho \sigma} b^{\rho} u^{\sigma}$.

We begin by noting that (B1d) is equivalent to (B1b). This can be shown by multiplying (B1d) by $\epsilon^{\alpha \beta \mu \nu}$ and using the identity

$$
\epsilon^{\alpha \beta \mu \nu} \epsilon_{\mu \nu \rho \sigma}=-2\left(\delta_{\rho}^{\alpha} \delta_{\sigma}^{\beta}-\delta_{\sigma}^{\alpha} \delta_{\rho}^{\beta}\right) .
$$

Below we show at the lowest order in gradient (B1a), that (B1c) and (B1d) are indeed satisfied by the following covariant solution:

$$
j^{\mu} \sim(u+b)^{\mu} \delta(p \cdot(u+b)) e^{-p_{T}^{2} / B} f(p \cdot u),
$$

with $p_{T}^{2}=-p^{2}+(p \cdot u)^{2}-(p \cdot b)^{2}$. We first see (B1a) and $(\mathrm{B} 1 \mathrm{c})$ are satisfied by the antisymmetry of the indices and on-shell conditions:

$$
\begin{aligned}
\Delta_{\mu} j^{\mu} & \sim \frac{\partial}{\partial p_{\nu}} B \epsilon_{\mu \nu \rho \sigma} b^{\rho} u^{\sigma}(u+b)^{\mu}=0, \\
p_{\mu} j^{\mu} \sim p \cdot(u+b) \delta(p \cdot(u+b)) & =0 .
\end{aligned}
$$

Equation (B1d) requires some work:

$$
\begin{aligned}
p^{\mu} j^{\nu} & -p^{\nu} j^{\mu}+\frac{1}{2} \epsilon^{\mu \nu \rho \sigma} \Delta_{\rho} j_{\sigma} \\
\sim & \left(p^{\mu}(u+b)^{\nu}-p^{\nu}(u+b)^{\mu}\right) \\
& \times \delta\left(p \cdot((u+b)) e^{-p_{T}^{2} / B} f(p \cdot u)\right. \\
+ & \frac{1}{2} \epsilon^{\mu \nu \rho \sigma} \frac{\partial}{\partial p_{\lambda}} B \epsilon_{\rho \sigma \alpha \beta} b^{\alpha} u^{\beta}(u+b)_{\sigma} \delta(p \cdot(u+b)) \\
& \times e^{-p_{T}^{2} / B} f(p \cdot u) .
\end{aligned}
$$

The second term can be simplified by noting that $\frac{\partial}{\partial p_{\lambda}}$ can pull out $p_{\lambda}, u_{\lambda}$, and $b_{\lambda}$. The last two cases always vanish when contracting with $\epsilon_{\rho \sigma \alpha \beta} b^{\alpha} u^{\beta}$. Keeping only the $p_{\lambda}$ contribution and using the following identity:

$$
\begin{aligned}
\epsilon^{\mu \nu \rho \sigma} \epsilon_{\rho \sigma \alpha \beta}= & -\left(\delta_{\lambda}^{\mu} \delta_{\alpha}^{\nu} \delta_{\beta}^{\sigma}+\delta_{\alpha}^{\mu} \delta_{\beta}^{\nu} \delta_{\lambda}^{\sigma}+\delta_{\beta}^{\mu} \delta_{\lambda}^{\nu} \delta_{\alpha}^{\sigma}\right. \\
& \left.-\delta_{\lambda}^{\mu} \delta_{\beta}^{\nu} \delta_{\alpha}^{\sigma}-\delta_{\beta}^{\mu} \delta_{\alpha}^{\nu} \delta_{\lambda}^{\sigma}-\delta_{\alpha}^{\mu} \delta_{\lambda}^{\nu} \delta_{\beta}^{\sigma}\right),
\end{aligned}
$$

we obtain from (B5)

$$
\sim p \cdot(u+b)\left(b^{\mu} u^{\nu}-b^{\nu} u^{\mu}\right) \delta(p \cdot(u+b)) e^{-p_{T}^{2} / B} f(p \cdot u),
$$

which vanishes by the on-shell condition.
[1] W. Heisenberg and H. Euler, Z. Phys. 98, 714 (1936).

[2] J. S. Schwinger, Phys. Rev. 82, 664 (1951).

[3] G. V. Dunne, in Heisenberg-Euler Effective Lagrangians: Basics and Extensions, edited by M. Shifman et al., From Fields to Strings Vol. 1 (World Scientific, Singapore, 2005), pp. 445-522.

[4] R. Baier and P. Breitenlohner, Nuovo Cimento B 47, 117 (1967).

[5] Z. Bialynicka-Birula and I. Bialynicki-Birula, Phys. Rev. D 2, 2341 (1970).

[6] S. L. Adler, Ann. Phys. (N.Y.) 67, 599 (1971).

[7] K. Hattori and K. Itakura, Ann. Phys. (Amsterdam) 330, 23 (2013).

[8] K. Hattori and K. Itakura, Ann. Phys. (Amsterdam) 334, 58 (2013).

[9] A. Vilenkin, Phys. Rev. D 22, 3080 (1980).

[10] D. Kharzeev, Phys. Lett. B 633, 260 (2006).

[11] D. Kharzeev and A. Zhitnitsky, Nucl. Phys. A797, 67 (2007).

[12] K. Fukushima, D. E. Kharzeev, and H. J. Warringa, Phys. Rev. D 78, 074033 (2008).

[13] M. A. Metlitski and A. R. Zhitnitsky, Phys. Rev. D 72, 045011 (2005).
[14] D. T. Son and A. R. Zhitnitsky, Phys. Rev. D 70, 074018 (2004).

[15] D. E. Kharzeev and H. U. Yee, Phys. Rev. D 83, 085007 (2011).

[16] D. T. Son and B. Z. Spivak, Phys. Rev. B 88, 104412 (2013).

[17] K. Hattori and D. Satow, Phys. Rev. D 94, 114032 (2016).

[18] K. Hattori, S. Li, D. Satow, and H. U. Yee, Phys. Rev. D 95, 076008 (2017).

[19] K. Fukushima and Y. Hidaka, Phys. Rev. Lett. 120, 162301 (2018).

[20] K. Fukushima and Y. Hidaka, J. High Energy Phys. 04 (2020) 162.

[21] W. Li, S. Lin, and J. Mei, Phys. Rev. D 98, 114014 (2018).

[22] S. Lin and L. Yang, Phys. Rev. D 101, 034006 (2020).

[23] N. Y. Astrakhantsev, V. V. Braguta, M. D'Elia, A. Y. Kotov, A. A. Nikolaev, and F. Sanfilippo, arXiv:1910.08516.

[24] R. Critelli, S. I. Finazzo, M. Zaniboni, and J. Noronha, Phys. Rev. D 90, 066006 (2014).

[25] S. Li and H. U. Yee, Phys. Rev. D 97, 056024 (2018).

[26] U. H. Danielsson and D. Grasso, Phys. Rev. D 52, 2533 (1995).

[27] J. Chao, L. Yu, and M. Huang, Phys. Rev. D 90, 045033 (2014); 91, 029903(E) (2015). 
[28] K. Fukushima, K. Hattori, H. U. Yee, and Y. Yin, Phys. Rev. D 93, 074028 (2016).

[29] J. Chao and M. Huang, arXiv:1609.04966.

[30] K. Hattori and D. Satow, Phys. Rev. D 97, 014023 (2018).

[31] B. Singh, S. Mazumder, and H. Mishra, J. High Energy Phys. 05 (2020) 068.

[32] D. T. Son and N. Yamamoto, Phys. Rev. Lett. 109, 181602 (2012).

[33] D. T. Son and N. Yamamoto, Phys. Rev. D 87, 085016 (2013).

[34] M. A. Stephanov and Y. Yin, Phys. Rev. Lett. 109, 162001 (2012).

[35] J. H. Gao, Z. T. Liang, S. Pu, Q. Wang, and X. N. Wang, Phys. Rev. Lett. 109, 232301 (2012).

[36] S. Pu, J.h. Gao, and Q. Wang, Phys. Rev. D 83, 094017 (2011).

[37] J. W. Chen, S. Pu, Q. Wang, and X. N. Wang, Phys. Rev. Lett. 110, 262301 (2013).

[38] Y. Hidaka, S. Pu, and D. L. Yang, Phys. Rev. D 95, 091901 (2017).

[39] C. Manuel and J. M. Torres-Rincon, Phys. Rev. D 89, 096002 (2014).

[40] C. Manuel and J. M. Torres-Rincon, Phys. Rev. D 90, 076007 (2014).

[41] Y. Wu, D. Hou, and H.c. Ren, Phys. Rev. D 96, 096015 (2017).

[42] N. Mueller and R. Venugopalan, Phys. Rev. D 96, 016023 (2017).

[43] N. Mueller and R. Venugopalan, Phys. Rev. D 97, 051901 (2018).

[44] A. Huang, S. Shi, Y. Jiang, J. Liao, and P. Zhuang, Phys. Rev. D 98, 036010 (2018).

[45] J. H. Gao, Z. T. Liang, Q. Wang, and X. N. Wang, Phys. Rev. D 98, 036019 (2018).

[46] S. Carignano, C. Manuel, and J. M. Torres-Rincon, Phys. Rev. D 98, 076005 (2018).

[47] S. Lin and A. Shukla, J. High Energy Phys. 06 (2019) 060.

[48] S. Carignano, C. Manuel, and J. M. Torres-Rincon, arXiv: 1908.00561.

[49] Y. C. Liu, L. L. Gao, K. Mameda, and X. G. Huang, Phys. Rev. D 99, 085014 (2019).

[50] Ö. F. Dayi and E. Kilinarslan, Phys. Rev. D 98, 081701 (2018).
[51] N. Weickgenannt, X. L. Sheng, E. Speranza, Q. Wang, and D. H. Rischke, Phys. Rev. D 100, 056018 (2019).

[52] J. H. Gao and Z.T. Liang, Phys. Rev. D 100, 056021 (2019).

[53] K. Hattori, Y. Hidaka, and D. L. Yang, Phys. Rev. D 100, 096011 (2019).

[54] Z. Wang, X. Guo, S. Shi, and P. Zhuang, Phys. Rev. D 100, 014015 (2019).

[55] J. H. Gao, Z. T. Liang, and Q. Wang, Phys. Rev. D 101, 096015 (2020).

[56] Y. C. Liu, K. Mameda, and X. G. Huang, arXiv:2002.03753.

[57] D. L. Yang, K. Hattori, and Y. Hidaka, arXiv:2002.02612.

[58] E. V. Gorbar, I. A. Shovkovy, S. Vilchinskii, I. Rudenok, A. Boyarsky, and O. Ruchayskiy, Phys. Rev. D 93, 105028 (2016).

[59] J. W. Chen, T. Ishii, S. Pu, and N. Yamamoto, Phys. Rev. D 93, 125023 (2016).

[60] Y. Hidaka, S. Pu, and D. L. Yang, Phys. Rev. D 97, 016004 (2018).

[61] N. Abbasi, F. Taghinavaz, and O. Tavakol, J. High Energy Phys. 03 (2019) 051.

[62] X. L. Sheng, R. H. Fang, Q. Wang, and D. H. Rischke, Phys. Rev. D 99, 056004 (2019).

[63] M. L. Bellac, Thermal Field Theory (Cambridge University Press, Cambridge, England, 2010).

[64] J. Gao, J. Pang, and Q. Wang, Phys. Rev. D 100, 016008 (2019).

[65] This is a consequence of the collisionless limit.

[66] K. Fukushima, Phys. Rev. D 83, 111501 (2011).

[67] Y. Akamatsu and N. Yamamoto, Phys. Rev. Lett. 111, 052002 (2013).

[68] The magnetic field in the boosted frame is $\sqrt{B^{2}-E^{2}}$, whose deviation from the background is negligible to linear order in $E_{M}$.

[69] J. Y. Chen, D. T. Son, M. A. Stephanov, H. U. Yee, and Y. Yin, Phys. Rev. Lett. 113, 182302 (2014).

[70] To linear order in the perturbation, the magnitude of the magnetic field $\sqrt{B^{2}+B_{\perp}^{2}}=B$ does not change. Only the direction changes.

[71] This is needed in order to satisfy (10). Redistribution of higher LL states also satisfies (10), but is energetically unfavored in the large $B$ limit. 